\title{
Test Statistics in Kalman Filtering
}

\author{
Jian-Guo Wang \\ Faculty of Science and Engineering, York University
}

\begin{abstract}
Many estimation problems can be modeled using a Kalman filter. One of the key requirements for Kalman filtering is to characterize various error sources, essentially for the quality assurance and quality control of a system. This characterization can be evaluated by applying the principle of multivariate statistics to the system innovations and the measurement residuals. This manuscript will systematically examine the test statistics in Kalman filter on the ground of the normal, $\chi^{2}-, \boldsymbol{t}$ - and $\boldsymbol{F}$ - distributions, and the strategies for global, regional and local statistical tests as well. It is hoped that these test statistics can generally help better understand and perform the statistical analysis in specific applications using a Kalman filter.
\end{abstract}

Key words: Kalman filter, test statistics, normal distribution, $\chi^{2}$ distribution, $\boldsymbol{t}$-distribution, $\boldsymbol{F}$-distribution

\section{Introduction}

Since 1980s, Geomatics professionals both in research and industry have increasingly shown their profound interest in applying the Kalman filter to various applications, such as kinematic positioning or navigation systems, image processing, and data processing of deformation monitoring etc. Undoubtedly, knowledge of Kalman filtering has become essential to the Geomatics researchers and professionals.

A Kalman filter is simply an optimal recursive data processing algorithm that blends all available information, including measurement outputs, prior knowledge about the system and measuring sensors, to estimate the state variables in such a manner that the error is statistically minimized [Maybeck, 1979]. In practice, linear equation system with white Gaussian noises is commonly taken as the standard model of a Kalman filter. However, one must generally face the following facts [Maybeck, 1979]: (1) no mathematical model is perfect, (2) dynamic systems are driven not only by own control inputs, but also by disturbances which can neither be controlled nor modelled deterministically, and (3) sensors do not provide perfect and complete data about a system.

Hence, a Kalman filter can function properly only if the assumptions about its model structures, dynamical process and measurement noise are correct or realistic. It can become divergent if any of the following situations occurs [Schlee et al, 1967; Tarn et al, 1970; Gelb, 1974; Stöhr, 1986; Loffeld, 1990]:

- Improper system model;

- False modeled process noise;

- False modeled measurement noise or

- Unexpected sudden changes of the state vectors

Correspondingly, one needs to study the behaviors of the errors associated with the system model. This may be called as system identification or system diagnostics, one of the advanced topics in Kalman filter.

There are different ways to perform system identification. Statistic tests belong to the essential methods of system identification. Herewith, the system model under the Null hypothesis is tested against one or multiple alternative hypotheses. The statistic algorithms can be divided into two categories. The first one is to make multiple hypotheses about the stochastic characteristics of a system (Multiple Hypothesis Filter Detectors) [Willsky, Deyst, Crawford, 1974, 1975; Willsky, 1976]. In order to reach a statistic decision, the posteriori probabilities of the state vectors will be calculated, for instance, through the sequential probability ratio test- SPRT [Willsky, 1976; Yoshimura, et al, 1979]. The second one is to perform the system identification with the help of series of system innovations ("Innovation-based detection") [Mehra, Peschon, 1971; Stöhr, 1986; Salzmann, Teunissen, 1989; etc.]. With this method, the signal is filtered using a normal model, until a failure is found through the statistic tests, for example, through GLR (Generalized likelihood ratio) method [Willsky, 1976; Huep, 1986] or more often through the specific test statistics based on normal, $\chi^{2}$, $\boldsymbol{t}$ - or $\boldsymbol{F}$ - distribution.

Since the Kalman filter was introduced, how to characterize the error sources of a system, especially the 
series of the system innovation, has caught certain research attention. [Stöhr, 1986] studied the statistic tests on the ground of Normal Distribution and $\chi^{2}$ Distribution using system innovation. [Salzmann, 1993] summarized a three-part test procedure as Detection, Identification and Adaptation (DIA) using system innovation for Kalman filter, in which the construction of test statistics is essential. [Wang, 1997] further discussed the test statistics not only on the basis of normal and $\chi^{2}$ distributions, but also on the basis of $t$ - and $F$ distributions using both of the system innovation and measurement residuals in Kalman filter.

A statistical test is no thing else, but a method of making statistical decisions based on the existing system model using experimental data. One needs statistic tests, for example, to identify abnormal dynamic changes of the system states, or to statistically verify the significance of the additional parameters, such as different sensor biases, in an integrated navigation system. Statistic tests can also help with studying the whiteness of system innovation. The detection of measurement outliers definitely needs statistic tests. A lot more examples exist in practice. They show how essential statistic tests are in Kalman filter so that a developer has to be capable of constructing the proper test statistics and applying them to practice.

However, there is still a lack of systematic description of fundamentals of test statistics for Kalman filter in textbooks. Most of the available works have missed out this topic or, if not the case, the test statistics is mostly based on the Normal Distribution and the $\chi^{2}$ Distribution only using system innovation. Applying of the $t$ - and $F$ - tests is not common.

From the perspective of both research and industry, it could be helpful to have a systematical understanding to the fundamentals of test statistics for Kalman filter, in order to use a Kalman filter well or develop new algorithms. However, the existing textbooks about Kalman filtering and applications do not normally talk about testing statistics, although they may be found miscellaneously in scientific publications of different fields. This manuscript aims to fill the gaps between the textbooks and scientific papers in the context of Kalman filter theory and applications.

This manuscript is organized as follows. The algorithm of Kalman filter is summarized in Section 2. Section 3 gives the estimation of the variance factor, or more precisely, the variance of unit weight. The statistic characteristics of filtering solutions are described in Section 4. Sections 5 and 6 are dedicated to building up various test statistics for system innovation and measurement residuals. The concluding remarks are given in the last section.

\section{ALGORITHM OF KALMAN FILTERING}

The Kalman filter is a set of mathematical equations that provide an efficient recursive means to estimate the state of a process through minimizing its mean squared errors. This section is to provide a brief introduction to the discrete Kalman filter, which includes its description and some discussion of the algorithm.

\subsection{The Model}

We consider a linear or linearized system with the statespace notation and assume that the data are available over a discrete time series $\left\{\boldsymbol{t}_{0}, \boldsymbol{t}_{1}, \ldots, \boldsymbol{t}_{N}\right\}$, which will often be simplified to $\{0,1, \ldots, N\}$. Without loss of generality, a deterministic system input vector will be droped in all of the expressions in this paper. Hence, at any time instant $\boldsymbol{k}(1 \leq \boldsymbol{k} \leq \boldsymbol{N})$ the system can be written as follows:

$$
\begin{aligned}
& x(k+1)=A(k+1, k) x(k)+B(k) w(k) \\
& z(k+1)=C(k+1) x(k+1)+\Delta(k+1)
\end{aligned}
$$

where $\boldsymbol{x}(\boldsymbol{k})$ is the $n$-dimensional state-vector, $z(\boldsymbol{k})$ is the $p$-dimensional observation vector, $\boldsymbol{w}(\boldsymbol{k})$ is the $m$ dimensional process noise vector, $\Delta(\boldsymbol{k})$ is the $p$ dimensional measurement noise vector, $\boldsymbol{A}(\boldsymbol{k}+1, \boldsymbol{k})$ is the $\boldsymbol{n} \times \boldsymbol{n}$ coefficient matrix of $\boldsymbol{x}(\boldsymbol{k}), \boldsymbol{B}(\boldsymbol{k})$ is the $\boldsymbol{n} \times \boldsymbol{m}$ coefficient matrix of $\boldsymbol{w}(\boldsymbol{k}), \boldsymbol{C}(\boldsymbol{k})$ is the $p \times n$ coefficient matrix of $\mathbf{z}(\boldsymbol{k})$. The random vectors $\boldsymbol{w}(\boldsymbol{k})$ and $\Delta(\boldsymbol{k})$ are generally assumed to be Gaussian with zeromean:

$$
\begin{aligned}
w(k) & \sim N(o, Q(k)) \\
\Delta(k) & \sim N(o, R(k))
\end{aligned}
$$

where $\boldsymbol{Q}(\boldsymbol{k})$ and $\boldsymbol{R}(\boldsymbol{k})$ are positive definite variance matrices, respectively. Further assumptions about the random noise are made and specified as follows $(\boldsymbol{i} \neq \boldsymbol{j})$ :

$$
\begin{aligned}
& \operatorname{Cov}(w(i), w(j))=O \\
& \operatorname{Cov}(\Delta(i), \Delta(j))=O \\
& \operatorname{Cov}(w(i), \Delta(j))=O
\end{aligned}
$$

Very often, we also have to assume the initial mean and variance-covariance matrix $\boldsymbol{x}(0)$ and $\boldsymbol{D}_{\boldsymbol{x} x}(0)$ for the system state at the time epoch 0 . In addition, the initial state $\boldsymbol{x}(0)$ is also assumed to be independent of $\boldsymbol{w}(\boldsymbol{k})$ and $\Delta(\boldsymbol{k})$ for all $\boldsymbol{k}$. 


\subsection{Kalman Filtering Equations}

To derive the optimal estimate $\hat{\boldsymbol{x}}(\boldsymbol{k})$ of $\boldsymbol{x}(\boldsymbol{k})$, one may use one of several optimality criterions to construct the optimal filter. For example, if the least-squares method is used, the optimality is defined in the sense of linear unbiased minimum variance, namely,

$$
\left.\begin{array}{l}
E\{\hat{x}\}=x \\
E\left\{(x-\hat{x})(x-\hat{x})^{T}\right\}=\min
\end{array}\right\}
$$

where $\hat{\boldsymbol{x}}$ is the unbiased minimum variance estimate of $\boldsymbol{x}$.

Under the given stochastic conditions in Section 2.1, one can derive the Kalman filtering for the state vector at $\boldsymbol{k}+1$ :

$$
\hat{\boldsymbol{x}}(\boldsymbol{k}+1)=\hat{\boldsymbol{x}}(\boldsymbol{k}+1 / \boldsymbol{k})+\boldsymbol{G}(\boldsymbol{k}+1) \boldsymbol{d}(\boldsymbol{k}+1 / \boldsymbol{k})
$$

and its variance-covariance matrix

$$
\begin{gathered}
\boldsymbol{D}_{x x}(\boldsymbol{k}+1)=\{\boldsymbol{E}-\boldsymbol{G}(\boldsymbol{k}+1) \boldsymbol{C}(\boldsymbol{k}+1)\} \boldsymbol{D}_{x x}(\boldsymbol{k}+1 / \boldsymbol{k}) \\
\left\{\boldsymbol{E}-\boldsymbol{G}(\boldsymbol{k}+1) \boldsymbol{C}(\boldsymbol{k}+1)+\boldsymbol{G}(\boldsymbol{k}+1) \boldsymbol{R}(\boldsymbol{k}+1) \boldsymbol{G}^{\boldsymbol{T}}(\boldsymbol{k}+1)\right.
\end{gathered}
$$

where

$$
\begin{aligned}
& \hat{\boldsymbol{x}}(\boldsymbol{k}+1 / \boldsymbol{k})=\boldsymbol{A}(\boldsymbol{k}+1, \boldsymbol{k}) \hat{\boldsymbol{x}}(\boldsymbol{k}) \\
& D_{x x}(k+1 / k)=A(k+1, k) D_{x x}(k) A^{T}(k+1, k) \\
& +B(k) Q(k) B^{T}(k) \\
& \boldsymbol{d}(\boldsymbol{k}+1 / \boldsymbol{k})=\mathbf{z}(\boldsymbol{k}+1)-\boldsymbol{C}(\boldsymbol{k}+1) \hat{\boldsymbol{x}}(\boldsymbol{k}+1 / \boldsymbol{k}) \\
& D_{d d}(k+1 / k)=C(k+1) D_{x x}(k+1 / k) C^{T}(k+1) \\
& +\boldsymbol{R}(\boldsymbol{k}+1) \\
& \boldsymbol{G}(\boldsymbol{k}+1)=\boldsymbol{D}_{x X}(\boldsymbol{k}+1 / \boldsymbol{k}) \boldsymbol{C}^{\boldsymbol{T}}(\boldsymbol{k}+1) \boldsymbol{D}_{d d}^{-1}(\boldsymbol{k}+1 / \boldsymbol{k})
\end{aligned}
$$

Here $\hat{\boldsymbol{x}}(\boldsymbol{k}+1 / \boldsymbol{k})$ is the one-step prediction of the state vector from the past epoch $k$ with its variance matrix $\boldsymbol{D}_{\boldsymbol{x} x}(\boldsymbol{k}+1 / \boldsymbol{k}), \boldsymbol{d}(\boldsymbol{k}+1 / \boldsymbol{k})$ is the system innovation vector with its variance matrix $\boldsymbol{D}_{\boldsymbol{d d}}(\boldsymbol{k}+1 / \boldsymbol{k})$, and $\boldsymbol{G}(\boldsymbol{k}+1)$ is the Kalman gain matrix.

An essential characteristic of the sequence $d(1 / 0), \ldots$, $\boldsymbol{d}(\boldsymbol{i}+1 / \boldsymbol{i}), \ldots, \boldsymbol{d}(\boldsymbol{k}+1 / \boldsymbol{k})$ is that they are independent from each other epochwise [Stöhr, 1986; Chui, Chen, 1987]:

$$
\operatorname{Cov}\{d(\boldsymbol{i}+1 / \boldsymbol{i}), \boldsymbol{d}(\boldsymbol{j}+1 / \boldsymbol{j})\}=\boldsymbol{O} \text { for }(\boldsymbol{i} \neq \boldsymbol{j})
$$

The stochastic characteristics of $\boldsymbol{d}(\boldsymbol{k}+1 / \boldsymbol{k})$ are obviously the mixture of the stochastic information from the real observation noise $\{\Delta(1), \Delta(2), \ldots\}$ and the system noise $\{\boldsymbol{w}(0), \boldsymbol{w}(1), \ldots\}$. Traditionally, the system innovation sequences are analyzed and used to build up the test statistics.

\subsection{An alternate Derivation of Kalman Filtering}

Let us analyze the error sources in Kalman filter in a different way. The optimal estimate $\hat{\boldsymbol{x}}(\boldsymbol{k}+1)$ of $\boldsymbol{x}(\boldsymbol{k}+1)$ at the instant $\boldsymbol{k}$ is always associated with the stochastic information, which may be divided into three independent groups:

a. The real observation noise $\Delta(\boldsymbol{k}+1)$,

b. The system noise $\boldsymbol{w}(\boldsymbol{k})$,

c. The noise from the predicted $\hat{\boldsymbol{x}}(\boldsymbol{k}+1 / \boldsymbol{k})$ through $\hat{\boldsymbol{x}}(\boldsymbol{k})$, on which the stochastic characteristics of $\{\Delta(1), \Delta(2), \ldots, \Delta(\boldsymbol{k})\},\{\boldsymbol{w}(0), \boldsymbol{w}(1), \ldots, \boldsymbol{w}(\boldsymbol{k}-1)\}$ are propagated through the system state model.

If these different error resources could be studied separately, it could be very helpful to evaluate the performance of a system in Kalman filter. Along with this line of thinking, the system model as in 2.1 can be reformulated through the three groups of the observation or residual equations as follows:

$$
\begin{aligned}
& \boldsymbol{v}_{\mathbf{l}_{x}}(\boldsymbol{k}+1)=\quad \hat{\boldsymbol{x}}(\boldsymbol{k}+1)-\boldsymbol{B}(\boldsymbol{k}) \hat{\boldsymbol{w}}(\boldsymbol{k})-\boldsymbol{l}_{\boldsymbol{x}}(\boldsymbol{k}+1) \\
& \boldsymbol{v}_{\boldsymbol{l}_{w}}(\boldsymbol{k}+1)=\quad \hat{\boldsymbol{w}}(\boldsymbol{k})-\boldsymbol{I}_{\boldsymbol{w}}(\boldsymbol{k}+1) \\
& v_{\boldsymbol{l}_{z}}(k+1)=C(k+1) \hat{x}(k+1) \quad-\boldsymbol{I}_{z}(k+1)
\end{aligned}
$$

where the independent (pseudo-)observation groups are simply listed by

$$
\begin{aligned}
& \boldsymbol{I}_{x}(\boldsymbol{k}+1)=A(\boldsymbol{k}+1, \boldsymbol{k}) \hat{\boldsymbol{x}}(\boldsymbol{k}) \\
& \boldsymbol{I}_{w}(\boldsymbol{k}+1)=w_{0}(\boldsymbol{k}) \\
& \boldsymbol{I}_{z}(\boldsymbol{k}+1)=\mathrm{z}(\boldsymbol{k}+1)
\end{aligned}
$$

with their variance-covariance matrices by

$$
\begin{aligned}
& D_{\boldsymbol{I}_{x} I_{x}}(k+1)=\boldsymbol{A}(\boldsymbol{k}+1, \boldsymbol{k}) \boldsymbol{D}_{x x}(\boldsymbol{k}) \boldsymbol{A}^{T}(\boldsymbol{k}+1, \boldsymbol{k}) \\
& \boldsymbol{D}_{\boldsymbol{I}_{w} \boldsymbol{I}_{w}}(\boldsymbol{k}+1)=\boldsymbol{Q}(\boldsymbol{k}) \\
& \boldsymbol{D}_{\boldsymbol{l}_{z} \boldsymbol{I}_{\mathrm{z}}}(\boldsymbol{k}+1)=\boldsymbol{R}(\boldsymbol{k}+1)
\end{aligned}
$$

$\boldsymbol{I}_{\boldsymbol{x}}(\boldsymbol{k}+1), \boldsymbol{I}_{\boldsymbol{w}}(\boldsymbol{k}+1)$ and $\boldsymbol{I}_{\boldsymbol{z}}(\boldsymbol{k}+1)=\mathbf{z}(\boldsymbol{k}+1)$ are the $\boldsymbol{n}$-, $\boldsymbol{m}$ - and $\boldsymbol{p}$-dimentional measurement or pseudomeasurement vectors, respectively. Usually $\boldsymbol{w}_{0}(\boldsymbol{k})=\boldsymbol{o}$. 
Again, by applying the least squares method, the identical estimate $\hat{\boldsymbol{x}}(\boldsymbol{k}+1)$ of $\boldsymbol{x}(\boldsymbol{k}+1)$ as in the section 2.2 can be obtained. For more details on this alternate derivation of Kalman filter and its advantages, the reader is referred to [Wang, 1997; Caspary and Wang, 1998].

This alternate derivation of Kalman filtering will directly make the measurement residual vectors available for error analysis and possibly to build up the test statistics in Kalman filter, since it is based on the measurement residual vectors. One can now analyse any of three measurement vectors through their own residual vectors. The measurement residual vectors are the functions of the system innovation vector epochwise

$$
\begin{aligned}
& v_{I_{x} I_{x}}(k)=D_{I_{x} I_{x}}(k) D_{x x}^{-1}(k / k-1) K(k) d(k / k-1) \\
& v_{I_{w} I_{w}}(k)=Q(k-1) B^{T}(k-1) D_{x x}^{-1}(k / k-1) K(k) d(k / k-1)
\end{aligned}
$$

$$
v_{l_{z} I_{z}}(k)=\{C(k) K(k)-E\} d(k / k-1)
$$

Similar to (16), we can readily prove the following results of independence:

$$
\operatorname{Cov}\{v(i), v(j)\}=O \text { for }(i \neq j)
$$

\section{VARIANCE OF WEIGHT UNIT}

The posteriori estimation of the variance of weight unit $\sigma_{0}^{2}$ is essential in Geomatics. Some confusion has been out there in applications of Kalman filer, because the variance-covariance matrices are directly used in Kalman filter. Surely the variance of weight unit, also called as variance factor, should be close to unity for a perfect model of system. However, this barely happens in practice.

An algorithm for the estimation of unknown variance factor $\sigma_{0}^{2}$ was constructed on the ground of the normalGamma distribution in [Koch, 1990]. It allows estimating the variance factor together with the state vector in Kalman filter. Alternatively, $\sigma_{0}^{2}$ can also be estimated by taking advantages of the sequences of the system innovation or the measurement residual vectors in [Wang, 1997 etc]. The single epoch estimate of $\sigma_{0}^{2}$, also called as the local variance of unit weight, is given by

$$
\hat{\sigma}_{l 0}^{2}(k)=\frac{v^{T}(k) D_{l l}^{-1}(k) v(k)}{r(k)}
$$

where

$$
v(k)=\left[v_{l_{x}}^{T}(k), v_{l_{w}}^{T}(k), v_{l_{z}}^{T}(k)\right]^{T}
$$

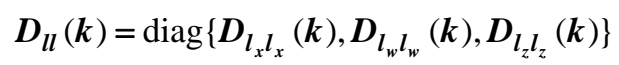

and $\boldsymbol{r}(\boldsymbol{k})$ is the number of the redundant measurements at epoch $k\left(t_{0}<t_{k} \leq t_{N}\right)$. An alternate expression exists:

$$
\hat{\sigma}_{l 0}^{2}(k)=\frac{d^{T}(k / k-1) D_{d d}^{-1}(k / k-1) d(k / k-1)}{r(k)}
$$

The proof of equivalence between (30) and (33) can be referred to [Pelzer, 1987; Tao, 1992; Wang, 1997]. One can also estimate the variance factor $\sigma_{0}^{2}$ over a specific time interval as the regional estimate of variance of unit weight. For example, over a certain specified time interval from epoch $(\boldsymbol{k}-\boldsymbol{s}+1)$ to epoch $\boldsymbol{k}$, one can order the system innovation for these $\boldsymbol{s}$ epochs as follows

$$
\begin{aligned}
d_{r}(\mathrm{~s})= & {\left[\boldsymbol{d}^{T}(\boldsymbol{k}+s-1 / k+s-2),\right.} \\
& \left.\boldsymbol{d}^{T}(\boldsymbol{k}+s-2 / \boldsymbol{k}+s-3), \ldots, \boldsymbol{d}^{T}(\boldsymbol{k} / \boldsymbol{k}-1)\right]^{\mathrm{T}}
\end{aligned}
$$

with its variance matrix

$$
\begin{aligned}
\boldsymbol{D}_{\boldsymbol{d}_{\boldsymbol{r}}(\mathrm{s}) \boldsymbol{d}_{\boldsymbol{r}}(\mathrm{s})}=\operatorname{diag}\left\{\boldsymbol{D}_{\boldsymbol{d d}}(\boldsymbol{k}+\boldsymbol{s}-1 / \boldsymbol{k}+\boldsymbol{s}-2),\right. \\
\\
\left.\boldsymbol{D}_{\boldsymbol{d d}}(\boldsymbol{k}+\boldsymbol{s}-2 / \boldsymbol{k}+\boldsymbol{s}-3), \ldots, \boldsymbol{D}_{\boldsymbol{d d}}(\boldsymbol{k} / \boldsymbol{k}-1)\right\}
\end{aligned}
$$

The regional estimate of $\sigma_{0}^{2}$ is then equal to

$\hat{\sigma}_{r 0}^{2}(k)=\frac{d^{T}(\mathrm{~s}) D_{d_{r}(s) d_{r}(s)}^{-1} d(\mathrm{~s})}{f_{r}(\mathrm{~s})}$

where $\boldsymbol{f}_{\boldsymbol{r}}$ (s) is the total number of the redundant measurements of $s$ epochs:

$$
f_{r}(s)=\sum_{j=1}^{s} r(k-s+j)
$$

Furthermore, the global estimate of $\sigma_{0}^{2}$ for all of the past $\boldsymbol{k}$ epochs can be calculated by

$\hat{\sigma}_{g 0}^{2}(k)=\frac{d_{g}^{T}(k) D_{d_{g}(k) d_{g}(k)}^{-1} d(k)}{f_{g}(k)}$

where

$\boldsymbol{d}_{\boldsymbol{g}}(\boldsymbol{k})=\left[\boldsymbol{d}^{T}(1 / 0), \boldsymbol{d}^{T}(2 / 1), \ldots, \boldsymbol{d}^{T}(\boldsymbol{k} / \boldsymbol{k}-1)\right]^{\mathrm{T}}$

$\boldsymbol{D}_{\boldsymbol{d}_{g}(\boldsymbol{k}) \boldsymbol{d}_{g}(\boldsymbol{k})}=\operatorname{diag}\left\{\boldsymbol{D}_{d d}(1 / 0), \boldsymbol{D}_{d d}(2 / 1), \ldots, \boldsymbol{D}_{d d}(\boldsymbol{k} / \boldsymbol{k}-1)\right\}$

$f_{g}(k)=\sum_{j=1}^{k} r(j)$ 


\section{STATISTIC CHARACTERISTICS OF FILTERING SOLUTIONS}

In order to evaluate the quality of the solutions and construct different test statistics, the statistic distributions of various random vectors are used in Kalman filter on the ground of the hypothesis: the normal distributed process and measurement noise as given in 3.1 will be discussed. At an arbitrary epoch $k$, all of the derived random variables or vectors are the functions of the measurement vector $I(k)=\left[I_{x}^{T}(k), I_{w}^{T}(k), I_{z}^{T}(k)\right]^{T}$ (see (20) $\left.\sim(22)\right)$. Among them, $\boldsymbol{d}(\boldsymbol{k} / \boldsymbol{k}-1), \hat{\boldsymbol{x}}(\boldsymbol{k}), \boldsymbol{v}(\boldsymbol{k}), \hat{\sigma}_{0}^{2}(\boldsymbol{k})$ are essential for quality control of a system. By applying the law of error propagation, their distributions are easily known as:

$$
\begin{aligned}
& \boldsymbol{d}(\boldsymbol{k} / \boldsymbol{k}-1) \sim N\left(0, D_{d d}(k / k-1)\right) \\
& \hat{x}(k) \sim N\left(\mu_{x}, D_{x x}(k)\right) \\
& v(k) \sim N\left(0, D_{v v}(k)\right) \\
& d^{T}(k / k-1) D_{d d}^{-1}(k / k-1) d(k / k-1) \\
& \quad=v^{T}(k) D_{I I}^{-1}(k) v(k) \sim \chi^{2}(r(k))
\end{aligned}
$$

where $\boldsymbol{N}(\boldsymbol{a}, \boldsymbol{b})$ represents a normal distribution with $\boldsymbol{a}$ and $\boldsymbol{b}$ as its expectation and variance, respectively.

The $i$-th component $\boldsymbol{d}_{\boldsymbol{i}}(\boldsymbol{k} / \boldsymbol{k}-1)$ in $\boldsymbol{d}(\boldsymbol{k} / \boldsymbol{k}-1)$ is normally distributed as follows:

$$
\begin{aligned}
\boldsymbol{d}_{\boldsymbol{i}}(\boldsymbol{k} / \boldsymbol{k}-1) \sim & \boldsymbol{N}\left(0, \sigma_{\boldsymbol{d}_{\boldsymbol{i}}(\boldsymbol{k} / \boldsymbol{k}-1)}^{2}\right) \\
& (i=1,2, \ldots, p ; k=1,2, \ldots N)
\end{aligned}
$$

Any arbitrary subvector of $\boldsymbol{d}(\boldsymbol{k} / \boldsymbol{k}-1)$ is also normally distributed. Based on the independency of the innovation vectors between two arbitrary epochs shown in (16), the vectors $\boldsymbol{d}_{\boldsymbol{r}}(\boldsymbol{s})$ and $\boldsymbol{d}_{\boldsymbol{g}}(\boldsymbol{k})$ as in (34) and (39) belong to the following normal distributions:

$$
\begin{aligned}
& d_{r}(s) \sim N\left(0, D_{d_{r}(s) d_{r}(s)}\right) \\
& d_{g}(k) \sim N\left(0, D_{d_{g}(k) d_{g}(k)}\right)
\end{aligned}
$$

wherein $\boldsymbol{D}_{\boldsymbol{d}_{\boldsymbol{r}}(\boldsymbol{s}) \boldsymbol{d}_{\boldsymbol{r}}(\boldsymbol{s})}$ and $\boldsymbol{D}_{\boldsymbol{d}_{\boldsymbol{g}}(\boldsymbol{k}) \boldsymbol{d}_{\boldsymbol{g}}(\boldsymbol{k})}$ are as in (35) and (40). Analog to (46), any arbitrary components $\boldsymbol{d}_{\boldsymbol{g i}}(\boldsymbol{k})$ and $\boldsymbol{d}_{\boldsymbol{r i}}(\boldsymbol{s})$ for the $\boldsymbol{i}$-th type of observations also belong to the normal distribution:

$$
\begin{array}{ll}
\boldsymbol{d}_{\boldsymbol{g i}}(\boldsymbol{k}) \sim \boldsymbol{N}\left(0, \boldsymbol{D}_{\boldsymbol{d}_{\boldsymbol{g i}}(\boldsymbol{k}) \boldsymbol{d}_{\boldsymbol{g i}}(\boldsymbol{k})}\right) & (i=1,2, \ldots, p) \\
\boldsymbol{d}_{\boldsymbol{r i}}(\boldsymbol{s}) \sim \boldsymbol{N}\left(0, \boldsymbol{D}_{\boldsymbol{d}_{\boldsymbol{r} i}(s) \boldsymbol{d}_{r i}(s)}\right) & (i=1,2, \ldots, p)
\end{array}
$$

where, for $i=1,2, \ldots, n+m+p$,

$$
\begin{gathered}
d_{g i}(k)=\left(d_{i}(1 / 0), \quad d_{i}(2 / 1), \quad \cdots, \quad d_{i}(k / k-1)\right)^{T} \\
d_{r i}(s)=\left(d_{i}(k-s+1 / k-s), \quad d_{i}(k-s+2 / k-s+1)\right. \\
\left.\cdots, \quad d_{i}(k / k-1)\right)^{T}
\end{gathered}
$$

with their corresponding variance-covariance matrices

$$
\begin{aligned}
& D_{d_{g i}(k) d_{g i}(k)}= \\
& \operatorname{diag}\left(\sigma_{d_{i}(1 / 0)}^{2}, \quad \sigma_{d_{i}(2 / 1)}^{2}, \cdots, \quad \sigma_{d_{i}(k / k-1)}^{2}\right) \\
& \boldsymbol{D}_{\boldsymbol{d}_{r i}(s) d_{r i}(s)}=\operatorname{diag}\left(\sigma_{d_{i}(k-s+1 / k-s)}^{2}, \quad \sigma_{d_{i}(k-s+2 / k-s+1)}^{2},\right. \\
& \left.\cdots, \quad \sigma_{d_{i}(k / k-1)}^{2}\right)
\end{aligned}
$$

The $i$-th component $\boldsymbol{v}_{\boldsymbol{i}}(\boldsymbol{k})$ of $\boldsymbol{v}(\boldsymbol{k})$ is of the normal distribution, too:

$$
\begin{aligned}
\boldsymbol{v}_{\boldsymbol{i}}(\boldsymbol{k}) \sim & \boldsymbol{N}\left(0, \sigma_{\boldsymbol{v}_{\boldsymbol{i}}(\boldsymbol{k})}^{2}\right) \\
& (i=1,2, \ldots, n+m+p ; k=1,2, \ldots, N)
\end{aligned}
$$

For any arbitrary subvector of $\boldsymbol{v}(\boldsymbol{k})$, e.g. $\boldsymbol{v}_{\boldsymbol{I}_{x}}(\boldsymbol{k}), \boldsymbol{v}_{\boldsymbol{l}_{w}}(\boldsymbol{k})$ or $\boldsymbol{v}_{\boldsymbol{l}_{\boldsymbol{z}}}(\boldsymbol{k})$, the normal distributions apply. The global cumulative measurement residual vector for all of the past epochs can be defined as

$v_{g}(k)=\left(v^{T}(1), \quad v^{T}(2), \quad \cdots, \quad v^{T}(k)\right)^{T}$

and the regional cumulative from the past $s$ epochs as

$v_{r}(s)=\left(\begin{array}{llll}v^{T}(k-s+1), & v^{T}(k-s+2), & \cdots, & v^{T}(k)\end{array}\right)^{T}$

with the following corresponding variance-covariance matrices

$$
\begin{aligned}
& D_{v_{g}(k) v_{g}(k)}= \\
& \operatorname{diag}\left(D_{v(1) v(1)}, \quad D_{v(2) v(2)}, \cdots, \quad D_{v(k) v(k)}\right) \\
& D_{v_{r}(s) v_{r}(s)}=\begin{aligned}
\operatorname{diag}\left(D_{v(k-s+1) v(k-s+1)},\right. & D_{v(k-s+2) v(k-s+2)}, \\
\ldots, & \left.D_{v(k) v(k)}\right)
\end{aligned}
\end{aligned}
$$

So $\boldsymbol{v}_{\boldsymbol{g}}(\boldsymbol{k})$ and $\boldsymbol{v}_{\boldsymbol{r}}(\boldsymbol{k})$ are obviously normally distributed:

$$
\begin{aligned}
& v_{g}(k) \sim N\left(0, D_{v_{g}(k) v_{g}(k)}\right) \\
& v_{r}(s) \sim N\left(0, D_{v_{r}(s) v_{r}(s)}\right)
\end{aligned}
$$

Similar to (49) and (50), the individual components of $\boldsymbol{v}_{\boldsymbol{g}}(\boldsymbol{k})$ and $\boldsymbol{v}_{\boldsymbol{r}}(\boldsymbol{k})$ : 


$$
\begin{gathered}
v_{g i}(k)=\left(v_{i}(1), \quad v_{i}(2), \quad \cdots, \quad v_{i}(k)\right)^{T} \\
v_{r i}(s)=\left(v_{i}(k-s+1), \quad v_{i}(k-s+2)\right. \\
\left.\ldots, \quad v_{i}(k)\right)^{T}
\end{gathered}
$$

belong to the following normal distributions

$$
\begin{gathered}
v_{g i}(k) \sim N\left(0, D_{v_{g i}(k) v_{g i}(k)}\right) \\
v_{r i}(s) \sim N\left(0, D_{v_{r i}(s) v_{r i}(s)}\right)
\end{gathered}
$$

with $i=1,2, \ldots, n+m+p$, where

$$
\begin{aligned}
& \boldsymbol{D}_{\boldsymbol{v}_{\boldsymbol{g} i}(\boldsymbol{k}) \boldsymbol{v}_{\boldsymbol{g} i}(\boldsymbol{k})}=\operatorname{diag}\left(\sigma_{\boldsymbol{v}_{\boldsymbol{i}}(1)}^{2}, \quad \sigma_{\boldsymbol{v}_{\boldsymbol{i}}(2)}^{2}, \cdots, \quad \sigma_{\boldsymbol{v}_{\boldsymbol{i}}(\boldsymbol{k})}^{2}\right) \\
& \boldsymbol{D}_{\boldsymbol{v}_{\boldsymbol{r} i}(s) \boldsymbol{v}_{\boldsymbol{r} i}(s)}=\operatorname{diag}\left(\sigma_{\boldsymbol{v}_{i}(k-s+1)}^{2}, \quad \sigma_{\boldsymbol{v}_{i}(k-s+2)}^{2}, \cdots, \quad \sigma_{v_{i}(k)}^{2}\right)
\end{aligned}
$$

are the variance matrices of $\boldsymbol{v}_{\boldsymbol{g i}}(\boldsymbol{k})$ and $\boldsymbol{v}_{\boldsymbol{r i}}(\boldsymbol{s})$.

For multiple components in $\boldsymbol{d}_{\boldsymbol{g}}(\boldsymbol{k}), \boldsymbol{d}_{\boldsymbol{r}}(\boldsymbol{s}), \boldsymbol{v}_{\boldsymbol{g}}(\boldsymbol{k})$ or $\boldsymbol{v}_{\boldsymbol{r}}(\boldsymbol{k})$, the same rule applies.

\section{TEST STATISTICS FOR SYSTEM INNOVATION}

This section will construct test statistics using system innovation. Under the assumption that no outliers exist in measurements, one could diagnose the possible failure caused by inappropriate state equations. Contrarily, one can identify the possible outliers under the assumption if the system model is assumed to be correct. The cause of a failure may be ambiguous and need to be analyzed in more details.

In this and next sections, we turn to perform statistic tests the epoch $\boldsymbol{k}=1,2, \ldots$ from the very beginning to an arbitrary epoch. The statistic tests will be introduced in three different levels, namely, global for all of the past $k$ epochs, regional for an arbitrary continuous epoch group, e.g., the $\boldsymbol{s}$ epochs in the past, and local for a single epoch (often the current epoch). The first two tests are very meaningful for the identification of systematic errors and the local one aims directly at the potential outliers or the unexpected sudden state changes.

\subsection{Global Test Statistics}

Global tests can be introduced in two different ways to investigate the system behaviors. Right after the first $k$ epochs are completed, one can perform the statistic tests with all of the system innovation information from the past and with their individual components by constructing the corresponding $\chi^{2}-$ test statistics.
With all of the past $k$ epochs together $(k=1,2, \ldots, N)$, the null hypothesis about $d_{g}(k)$

$\boldsymbol{H}_{0}: \boldsymbol{d}_{\boldsymbol{g}}(\boldsymbol{k})=0$ or $\boldsymbol{H}_{0}: \boldsymbol{E}\left(\chi_{\boldsymbol{d}_{\boldsymbol{g}}(\boldsymbol{k})}^{2}\right)=\tilde{\sigma}_{0}^{2}=1.0$

and its alternative

$$
\boldsymbol{H}_{1}: \boldsymbol{d}_{\boldsymbol{g}}(\boldsymbol{k}) \neq 0 \text { or } \boldsymbol{H}_{1}: \boldsymbol{E}\left(\chi_{\boldsymbol{d}_{\boldsymbol{g}}(\boldsymbol{k})}^{2}\right) \neq \tilde{\sigma}_{0}^{2}=1.0
$$

can be performed according to (48) by using the test statistic [Salzmann, Teunissen, 1989]

$\chi_{\boldsymbol{d}_{g}(k)}^{2}=d_{g}^{T}(k) D_{d_{g}(k) d_{g}(k)}^{-1} d_{g}(k) \sim \chi^{2}\left(\alpha_{g}, f_{g}(k)\right)$

at a significance level with the Type I error $\alpha_{\boldsymbol{g}}$. $\chi^{2}(\alpha, \boldsymbol{f})$ is the $(1-\alpha)$ - critical value from $\chi^{2}-$ Distribution with the degrees of freedom of $f$ after (41). The null hypothesis (68) will be rejected if

$\chi_{\boldsymbol{d}_{\boldsymbol{g}}(\boldsymbol{k})}^{2}>\chi^{2}\left(\alpha_{\boldsymbol{g}}, \boldsymbol{f}_{\boldsymbol{g}}(\boldsymbol{k})\right)$

The test can easily be extended to the $i$-th component $\boldsymbol{d}_{\boldsymbol{g} \boldsymbol{i}}(\boldsymbol{k})$ in $\boldsymbol{d}_{\boldsymbol{g}}(\boldsymbol{k})$ for $i=1,2, \ldots, p$ and $k=1,2, \ldots, N$. Under the null hypothesis

$H_{0}: d_{g i}(k)=0$

against its alternative

$$
H_{1}: d_{g i}(k) \neq 0
$$

Based on (49), the test statistic can be given by

$$
\begin{aligned}
& \chi_{\boldsymbol{d}_{\boldsymbol{g i}}(\boldsymbol{k})}^{2}=\boldsymbol{d}_{\boldsymbol{g} \boldsymbol{i}}^{\boldsymbol{T}}(\boldsymbol{k}) \boldsymbol{D}_{\boldsymbol{d}_{\boldsymbol{g} i}(\boldsymbol{k}) \boldsymbol{d}_{\boldsymbol{g} i}(\boldsymbol{k})}^{-1} \boldsymbol{d}_{\boldsymbol{g} \boldsymbol{i}}(\boldsymbol{k}) \sim \chi^{2}(\boldsymbol{k}) \\
& \text { If } \\
& \chi_{\boldsymbol{d}_{\boldsymbol{g} i}(\boldsymbol{k})}^{2}>\chi^{2}\left(\alpha_{\boldsymbol{g i}}, \boldsymbol{k}\right) \quad(i=1, \ldots, p)
\end{aligned}
$$

under the given significance level $\alpha_{g \boldsymbol{i}}$, the null hypothesis will be rejected.

\subsection{Regional Tests}

For the regional system diagnose, the processed $k$ epochs can be grouped at the user's wish. Without loss of the generality, the discussion here will be limited to two groups. We consider having the first group for the first $k-$ $s$ epochs (from 1 to epoch $k-s$ ) and the second group for 
the rest of the epochs (from epoch $k-s+1$ to epoch $k$ ) as $\boldsymbol{d}_{\boldsymbol{r}}(\boldsymbol{k}-\boldsymbol{s})$ (equivalent to $\boldsymbol{d}_{\boldsymbol{g}}(\boldsymbol{k}-\boldsymbol{s})$ ) and $\boldsymbol{d}_{\boldsymbol{r}}(\boldsymbol{s})$.

The null hypothesis about $\boldsymbol{d}_{\boldsymbol{r}}(\boldsymbol{s})$ is

$$
H_{0}: d_{r}(s)=1.0
$$

against the alternative

$$
H_{1}: d_{r}(s) \neq 0
$$

On the ground of the test statistic [Willsky, 1976; Stöhr, 1986; Salzmann, Teunissen, 1989], the following test is performed

$$
\chi_{d_{r}(s)}^{2}=d_{r}^{T}(s) D_{d_{r}(s) d_{r}(s)}^{-1} d_{r}(s) \sim \chi^{2}\left(f_{r}(s)\right)
$$

at the significance level of $\alpha_{r}$, where the number $\boldsymbol{f}_{\boldsymbol{r}}(\boldsymbol{s})$ is the degrees of freedom as in (37). For the second group $\boldsymbol{d}_{\boldsymbol{r}}(\boldsymbol{k}-\boldsymbol{s})$ also has the $\chi^{2}$ - distribution as

$$
\begin{gathered}
\chi_{d_{g}(k-s)}^{2}=d_{g}^{T}(k-s) D_{d_{g}(k-s) d_{g}(k-s)}^{-1} d_{g}(k-s) \\
\sim \chi^{2}\left(f_{g}(k-s)\right)
\end{gathered}
$$

An additional $F$-test statistic can be constructed to test the variance homogeneity between (78) and (79) or (70) because $\boldsymbol{d}_{\boldsymbol{r}}(\boldsymbol{s})$ is independent from $\boldsymbol{d}_{\boldsymbol{r}}(\boldsymbol{k}-\boldsymbol{s})$. This $F$ Test is given by

$$
\begin{aligned}
& F_{d_{r}(s)}=\frac{\hat{\sigma}_{r 0}^{2}(s)}{\hat{\sigma}_{g 0}^{2}(k-s)} \sim F\left(f_{r}(s), f_{g}(k-s)\right) \\
& \text { with } \quad \hat{\sigma}_{r 0}^{2}(s)=\frac{d_{r}^{T}(s) D_{d_{r}(s) d_{r}(s)}^{-1} d_{r}(s)}{f_{r}(s)} \\
& \hat{\sigma}_{g 0}^{2}(k-s)=\frac{d_{g}^{T}(k-s) D_{d_{g}(k-s) d_{r}(k-s)}^{-1} d_{g}(k-s)}{f_{g}(k-s)}
\end{aligned}
$$

$\boldsymbol{F}(\boldsymbol{a}, \boldsymbol{b})$ in (80) is the critical value of the Fisher distribution with the $1^{\text {st }}$ degrees of freedom $\boldsymbol{a}$ for the numerator and the $2^{\text {nd }}$ one $\boldsymbol{b}$ for the denominator. This test is always one-sided under a user- specified Type I error $\alpha$ as the significance level. An exchange between the numerator and the denominator may need in case $\hat{\sigma}_{\boldsymbol{g} 0}^{2}(\boldsymbol{k}-\boldsymbol{s})$ greater than $\hat{\sigma}_{\boldsymbol{r} 0}^{2}(\boldsymbol{s})$. This test is commonly employed to diagnose the significant difference between the first $k-s$ epochs and the rest of $s$ epochs.

For the $i$-th component $\boldsymbol{d}_{\boldsymbol{r i}}(\boldsymbol{s})$ in $\boldsymbol{d}_{\boldsymbol{r}}(\boldsymbol{s})$, one can also construct a $\chi^{2}$ test based on (50) and another $\boldsymbol{F}$-test analogue to (80). It runs
$H_{0}: d_{r i}(s)=0$

against the alternative

$$
H_{1}: d_{r i}(s) \neq 0
$$

by using the $\chi^{2}$ test statistics

$\chi_{d_{r i}(s)}^{2}=d_{r i}^{T}(s) D_{d_{r i}(s) d_{r i}(s)}^{-1} d_{r i}(s) \sim \chi^{2}(s)$

An $\boldsymbol{F}$-test runs for their variance homogeneity between (85) and (79) or (70) as follows

$F_{d_{g i}(s)}=\frac{d_{r i}^{T}(s) D_{d_{r i}(s) d_{r i}(s)}^{-1} d_{r i}(s) / s}{\hat{\sigma}_{g 0}^{2}(k-s)} \sim F\left(s, f_{g}(k-s)\right)$

for $i=1, \ldots, p$ at the significant level of $\alpha_{r i}$.

\subsection{Local Tests}

Through the local system diagnose, the tests can be introduced for the innovation vector as a whole and for its components, respectively.

At an arbitrary epoch $k$, the null hypothesis for $\boldsymbol{d}(\boldsymbol{k} / \boldsymbol{k}-1)$

$\boldsymbol{H}_{0}: \boldsymbol{d}(\boldsymbol{k} / \boldsymbol{k}-1)=0$

against the alternative

$$
\boldsymbol{H}_{0}: \boldsymbol{d}(\boldsymbol{k} / \boldsymbol{k}-1) \neq 0
$$

can be given. Its test statistic runs

$$
\begin{aligned}
\chi_{\boldsymbol{d}(\boldsymbol{k} / \boldsymbol{k}-1)}^{2}= & \boldsymbol{d}^{T}(\boldsymbol{k} / \boldsymbol{k}-1) \boldsymbol{D}_{\boldsymbol{d d}}^{-1}(\boldsymbol{k} / \boldsymbol{k}-1) \boldsymbol{d}(\boldsymbol{k} / \boldsymbol{k}-1) \\
& \sim \chi^{2}(\boldsymbol{r}(\boldsymbol{k}))
\end{aligned}
$$

at the significance level of $\alpha_{l}$ with the degrees of freedom $r(k)$.

A further $\boldsymbol{F}$-test statistic can be introduced as

$$
\boldsymbol{F}_{\boldsymbol{d}(\boldsymbol{k} / \boldsymbol{k}-1)}=\frac{\hat{\sigma}_{0}^{2}(\boldsymbol{k})}{\hat{\sigma}_{\boldsymbol{r} 0}^{2}(\boldsymbol{s})} \sim \boldsymbol{F}\left(\boldsymbol{r}(\boldsymbol{k}), \boldsymbol{f}_{\boldsymbol{r}}(\boldsymbol{s})\right)(k=2,3, \ldots, N)
$$

for the variance homogeneity between (81) and (33) or (88). $s$ means arbitrary specific epochs between epoch 1 and epoch $k-1$.

The quadratic form in (45) contains the entire information from the system innovation for an arbitrary epoch. Therefore, the causes of a system failure must be localized after the rejection of a $\chi_{\boldsymbol{d}(\boldsymbol{k} / \boldsymbol{k}-1)}^{2}$ or $\boldsymbol{F}_{\boldsymbol{d}(\boldsymbol{k} / \boldsymbol{k}-1)}$ test. It should orient to the individual error sources, e.g. 
the individual measurements or the individual process noise factors etc. in kinematic positioning or navigation. One should perform the further statistic tests for the individual measurements.

In order to perform the statistic tests for multiple components in $\boldsymbol{d}(\boldsymbol{k} / \boldsymbol{k}-1)$, the method for detection of position displacements in deformation analysis can be employed. More on this can be found in [Chrzanowski, Chen, 1986].

The test statistic for single component of $\boldsymbol{d}(\boldsymbol{k} / \boldsymbol{k}-1)$ can directly be constructed on the ground of the normal distribution or the $t$-distribution. The null hypothesis is

$$
\boldsymbol{H}_{0}: \boldsymbol{E}\left(\boldsymbol{d}_{\boldsymbol{i}}(\boldsymbol{k} / \boldsymbol{k}-1)\right)=0
$$

with its alternative

$$
\boldsymbol{H}_{1}: \boldsymbol{E}\left(\boldsymbol{d}_{\boldsymbol{i}}(\boldsymbol{k} / \boldsymbol{k}-1)\right) \neq 0
$$

for $i=1,2, \ldots, p$ and $k=1,2, \ldots, N$. According to (46) the test is performed

$$
N_{d_{i}(k / k-1)}=\frac{d_{i}(k / k-1)}{\sigma_{d_{i}(k / k-1)}} \sim N(0,1)
$$

at the significant level of $\alpha_{l i}$. The null hypothesis will be accepted if the two-sided test satisfies

$$
\begin{aligned}
&-\boldsymbol{u}_{1-\frac{\alpha_{l i}}{2}} \leq \frac{\boldsymbol{d}_{\boldsymbol{i}}(\boldsymbol{k} / \boldsymbol{k}-1)}{\sigma_{\boldsymbol{d}_{\boldsymbol{i}}(\boldsymbol{k} / \boldsymbol{k}-1)}} \leq \boldsymbol{u}_{1-\frac{\alpha_{l i}}{2}} \\
&(i=1,2, \ldots, p ; k=1,2, \ldots, N)
\end{aligned}
$$

where $\boldsymbol{u}_{1-\frac{\alpha_{\mathbf{l i}}}{2}}$ is a $\left(1-\frac{\alpha_{\boldsymbol{l i}}}{2}\right)$-critical value from the standard normal distribution. Furthermore, based on the past system information, (93) can be extended to the following $t$-test

$$
\begin{gathered}
\boldsymbol{T}_{\boldsymbol{d}_{\boldsymbol{i}}(\boldsymbol{k} / \boldsymbol{k}-1)}=\frac{\boldsymbol{d}_{\boldsymbol{i}}(\boldsymbol{k} / \boldsymbol{k}-1) / \sigma_{\boldsymbol{d}_{\boldsymbol{i}}(\boldsymbol{k} / \boldsymbol{k}-1)}}{\hat{\sigma}_{\boldsymbol{r} 0}(\boldsymbol{s})} \sim \boldsymbol{t}\left(\boldsymbol{f}_{\boldsymbol{r}}(\boldsymbol{s})\right) \\
(i=1,2, \ldots, p ; k=2,3, \ldots, N)
\end{gathered}
$$

The most common case is to test the current epoch $\boldsymbol{k}$ vs. the past $k-1$ epochs.

The differences between (93)-(94) and (88)-(89) are obvious. However, which one is preferable will absolutely depend on the user. A $\boldsymbol{t}$-test or an $\boldsymbol{F}$-test may deliver the more reliable results of fit to the real data, while a normal or a $\chi^{2}$ test is introduced with respect to the a-priori assumption.

\section{TEST STATISTICS FOR MEASUREMENT RESIDUALS}

As it can be seen, the system innovation mixes up different types of information. But it is transferred to the individual measurement residuals epoch by epoch through (26) (28), i.e., the residual vector $\boldsymbol{v}_{\boldsymbol{l}_{\boldsymbol{x}}}(\boldsymbol{k})$ for the predicted state vector, the residual vector $\boldsymbol{v}_{\boldsymbol{I}_{\boldsymbol{w}}}(\boldsymbol{k})$ for the process noise and the residual vector $\boldsymbol{v}_{\boldsymbol{l}_{\boldsymbol{z}}}(\boldsymbol{k})$ for the direct measurements. In this way, these different types of random information can separately be studied. On the basis of the fact that (30) and (33) are equivalent, the test statistics $\chi_{\boldsymbol{d}_{\boldsymbol{g}}(\boldsymbol{k})}^{2}, \chi_{\boldsymbol{d}_{\boldsymbol{r}}(\boldsymbol{s})}^{2}$ and $\chi_{\boldsymbol{d}(\boldsymbol{k} / \boldsymbol{k}-1)}^{2}$ in (70), (78) and (88) can also be derived using the measurement residual vector $\boldsymbol{v}(\boldsymbol{k})$. But it is not necessary to be repeated here. Therefore, only the test statistics for the individual components will be discussed in this section.

\subsection{Global Tests}

For the $i$-th component $\boldsymbol{v}_{\boldsymbol{g i}}(\boldsymbol{k})$ in $\boldsymbol{v}_{\boldsymbol{g}}(\boldsymbol{k})$, the null hypothesis is

$$
\boldsymbol{H}_{0}: \boldsymbol{v}_{\boldsymbol{g i}}(\boldsymbol{k})=0 \quad(i=1,2, \ldots, n+m+p)
$$

against the alternative

$$
H_{1}: v_{g i}(k) \neq 0
$$

The test statistic is given by

$$
\chi_{\boldsymbol{v}_{g i}(k)}^{2}=\boldsymbol{v}_{g i}^{\boldsymbol{T}}(\boldsymbol{k}) \boldsymbol{D}_{\boldsymbol{v}_{g i}(k) v_{g i}(k)}^{-1} \boldsymbol{v}_{g i}(k) \sim \chi^{2}(k)
$$

with the degrees of freedom of $k$. The null hypothesis will be rejected if

$$
\chi_{V_{g i}(k)}^{2}>\chi^{2}(\boldsymbol{k}) \quad(i=1,2, \ldots, n+m+p)
$$

at the significant level of $\bar{\alpha}_{g i}$.

\subsection{Regional Tests}

For the $i$-th component $v_{r i}(s)$ from $v_{r}(s)$, a $\chi^{2}$-test and a $F$-test can be constructed. The null hypothesis is

$H_{0}: v_{r i}(s)=0$

with the alternative

$$
H_{1}: v_{r i}(s) \neq 0
$$

The corresponding test statistic is given by

$$
\begin{array}{r}
\chi_{v_{r i}(s)}^{2}=v_{r i}^{T}(s) D_{v_{r i}(s) v_{r i}(s)}^{-1} v_{r i}(s) \sim \chi^{2}(s) \\
(i=1,2, \ldots, n+m+p)
\end{array}
$$


with the degrees of freedom of $s$. For the variance homogeneity between the independent $\chi_{v_{r i}(s)}^{2}$ and $\chi_{\boldsymbol{d}_{\boldsymbol{g}}(\boldsymbol{k}-\boldsymbol{s})}^{2}$ similar to (80), the F-test statistic can be introduced as

$$
F_{v_{g i}(s)}=\frac{v_{r i}^{T}(s) D_{v_{r i}}^{-1}(s) v_{r i}(s)}{v_{r i}(s) / s} \sim F\left(s, f_{g}(k-s)\right)
$$

\subsection{Local Tests}

The single outlier detection at a single epoch can be modeled through the null hypothesis

$$
\boldsymbol{H}_{0}: \boldsymbol{v}_{\boldsymbol{i}}(\boldsymbol{k})=0
$$

against the alternative

$$
H_{1}: v_{i}(k) \neq 0
$$

for $i=1,2, \ldots, m+n+p$ and $k=1,2, \ldots$ after the test of its standardized residual

$$
N_{v_{i}(k)}=\frac{v_{i}(k)}{\sigma_{v_{i}(k)}} \sim N(0,1)
$$

at the significant level of $\bar{\alpha}_{\boldsymbol{l}}$. The null hypothesis (102) will be accepted if

$$
\begin{aligned}
-\boldsymbol{u}_{1-\frac{\bar{\alpha}_{l i}}{2}} \leq & \frac{\boldsymbol{v}_{\boldsymbol{i}}(\boldsymbol{k})}{\sigma_{\boldsymbol{v}_{\boldsymbol{i}}(\boldsymbol{k})}} \leq \boldsymbol{u}_{1-\frac{\bar{\alpha}_{l i}}{2}} \\
& (i=1,2, \ldots, m+n+p ; k=1,2, \ldots, N)
\end{aligned}
$$

Besides a $t$-test between (105) and (78) or (70) can be introduced as follows

$$
\begin{aligned}
\boldsymbol{T}_{\boldsymbol{v}_{\boldsymbol{i}}(\boldsymbol{k})}= & \frac{\boldsymbol{v}_{\boldsymbol{i}}(\boldsymbol{k}) / \hat{\sigma}_{\boldsymbol{v}_{\boldsymbol{i}}(\boldsymbol{k})}}{\sigma_{\boldsymbol{r} 0}(\boldsymbol{s})} \sim \boldsymbol{t}\left(\boldsymbol{f}_{\boldsymbol{r}}(\boldsymbol{s})\right) \\
& (i=1,2, \ldots, n+m+p ; k=2, \ldots, N)
\end{aligned}
$$

For any epoch $k$, one can use $\boldsymbol{v}_{\boldsymbol{l}_{\boldsymbol{x}}}(\boldsymbol{k}), \boldsymbol{v}_{\boldsymbol{I}_{\boldsymbol{w}}}(\boldsymbol{k})$ and $\boldsymbol{v}_{\boldsymbol{l}_{\boldsymbol{z}}}(\boldsymbol{k})$, along with $\boldsymbol{v}(\boldsymbol{k})$ to investigate the statistic characteristics of measurement vectors $\boldsymbol{I}_{\boldsymbol{x}}(\boldsymbol{k}), \boldsymbol{I}_{\boldsymbol{w}}(\boldsymbol{k})$ and $\boldsymbol{l}_{\boldsymbol{z}}(\boldsymbol{k})$, especially two latter ones. Multiple components are possibly diagnosed together in the same way as mentioned in 5.3.

\section{CONCLUDING REMARKS}

Based on the standard model of Kalman filter, different test statistics have been elaborated on the basis of the normal, $\chi^{2}$-, $\boldsymbol{t}$ - and $\boldsymbol{F}$-distributions in this manuscript. This work can be conducive to better understanding of the statistic fundamentals in Kalman filter, provides some insights into statistic testing methods and applications.

In particular, the system innovation vector is transformed to the residual vectors of three measurement and pseudomeasurement groups by the aid of an alternative derivation of Kalman filter algorithm. This makes possible to construct test statistics directly using the measurement residual vectors so that the system diagnosis can directly aim at different error sources of interests to users. The given posteriori estimate of variance of weight unit in Section 3 can be used either to scale the variance and covariance matrices, or to reveal the difference between the model and the processed data set. On the ground of statistic characteristics of filter solutions summarized in the section 4 , the test statistics using the series of system innovation are constructed in the section 5 globally with $\chi^{2}$ - test, regionally either with $\chi^{2}$ - test or $\boldsymbol{F}$ - test, and locally either with $\boldsymbol{t}$ - test or the normal test according to the normal distribution. Analogous to the section 5 , the section 6 constructs the corresponding test statistics using the measurement residuals. Fortunately, $\chi^{2}$ - test, $\boldsymbol{F}$ - test, $\boldsymbol{t}$ - test and the normal test are four most commonly used statistic tests. The choice between a $\chi^{2}$ - test and a $\boldsymbol{F}$ - test, or between a $\boldsymbol{t}$ - test and a normal test, wherever two parallel tests are available, is left to the user.

How to construct a test statistics is more or less a theoretical task. But how to efficiently design the procedures to introduce the statistic tests in practice mostly depends on the understanding about the theory and the application. Practical experience plays an essential role in helping deliver a realistic and reliable test scheme. This manuscript however has limited to the testing statistics for general purposes instead of a specific application.

\section{References}

Caspary, Wilhelm; Wang, Jianguo (1998). Redundanzanteile und Varianzkomponenten im Kalman Filter. Vol.123, No.4, 1998, pp.121-128.

Chrzanowski, A.; Chen, Y. Q. (1986). Report on the Ad Hoc Committee on the Analysis of Deformation Surveys. XVIII International Congress FIG, Toronto, Canada, 1 11 June 1986, pp. 166-185.

Gelb, Arthur et al. (1974). Applied Optimal Estimation. The Analytic Sciences Corporation \& The M.I.T. Press, Cambridge Massachusetts London, 1974. 
Huep, Wolfgang (1986). Zur Positionsschätzung im gestörten Kalman-Filter am Beispiel eines manövrierenden Wasserfahrzeuges. Dissertation, Wissenschaftliche Arbeiten der Fachrichtung Vermessungswesen der Universität Hannover, Heft 143, Hannover, 1986.

Koch, K. Rudolf (1990). Bayesian Inference with Geodetic Applications, $2^{\text {nd }}$ edition, Springer-Verlag, Berlin, 1990.

Loffeld, Otmar. (1990). Estimationstheorie II, Anwendung-Kalman-Filter. R. Oldenbourg Verlag, München Wien, 1990.

Maybeck, Peter S. (1979). Stochastic models, estimation, and control. Volume 1, Academic Press, New York, 1979.

Mehra, R. K.; Peschon, J. (1971). An Innovations Approach to Fault Detection and Diagnosis in Dynamic Systems. Automatica, Vol. 16, 1971, S. 637640.

Pelzer, Hans (1987). Deformationsuntersuchungen auf der Basis kinematischer Bewegungsmodelle. AVN, Year 94, No. 2, February 1987, pp. 49 62.

Salzmann, Martin; Teunissen, P.J.G. (1989). Quality Control in Kinematic Data Processing. Land Vehicle Navigation 1989, DGON, Verlag TÜV Rheinland, Köln, 1989, pp. 355-366.

Salzmann, Martin (1993). Least Squares Filtering and Testing for Geodetic Navigation Applications. Number 37, Publication on Geodesy, Netherlands Geodetic Commission, Delft, 1993.

Schlee, F. H.; Standish, C. J.; et al (1967). Divergence in the Kalman Filter. AIAA Journal, Vol. 5, No. 6, June 1967, pp.1114-1120.
Stöhr, M. (1986). Der Kalman-Filter und seine Fehlerprozeße unter besonderer Berücksichtigung der Auswirkung von Modellfehlern. ForschungAusbildung-Weiterbildung Bericht Heft 19, Universität Kaiserlautern, Fachbereich Mathematik, August 1986.

Tao, Benzao (1992). Statistic Analysis of Measurements. Publishing house of Surveying and Mapping, Beijing, June 1992.

Tarn, T. J.; Zaborszky, J. (1970). A Practical, Nondiverging Filter. AIAA Journal, Vol. 8, No. 6, June 1970, pp.1127-1133.

Wang, Jianguo (1997). Filtermethoden zur fehlertoleranten kinematischen Positionsbestimmung. Schritenreihe Studiengang Vermessungswesen, Federal Arm-Forced University Munich, Germany, No. 52, Neubiberg, 1997.

Willsky, Alan S., Deyst, J. J.; Crawford, B. S. (1974). Adaptive Filtering and Self-Test Methods for Failure Detection and compensation. Proceedings of the 1974 JACC, Austin, Texas, June 19-21, 1974.

Willsky, Alan S., Deyst, J. J.; Crawford, B. S. (1975). Two Self-Test Methods Applied to an Inertial System Problem. Journal of Spacecraft, Vol. 12, No. 7, July 1975, S. 434-437.

Willsky, Alan S. (1976). A Survey of Design Methods for Failure Detection in Dynamic Systems. Automatica, Vol. 12, 1976, pp. 601-611.

Yoshimura, Toshio; et al. (1979). A Sequential Failure Detection Approach and the Identification of Failure Parameters. International Journal of System Science, Vol. 10, No. 7, 1979, S. 827 -836. 